\title{
The Role of Radical Surgery in the Management of Acquired Uterine Arteriovenous Malformation
}

\author{
Janelle K. Moulder ${ }^{a} \quad$ Leslie A. Garrett ${ }^{\mathrm{a}} \quad$ Gloria M. Salazar ${ }^{\mathrm{b}}$ \\ Annekathryn Goodman ${ }^{\text {a }}$ \\ Departments of ${ }^{\mathrm{a}}$ Obstetrics and Gynecology and ${ }^{\mathrm{b}}$ Interventional Radiology, \\ Massachusetts General Hospital, Boston, Mass., USA
}

\section{Key Words}

Arteriovenous fistula · Arteriovenous malformation · Cesarean scar pregnancy · Hemorrhage

\begin{abstract}
Background: Acquired arteriovenous malformations (AVMs) can develop after uterine instrumentation. The increased risks of vascular changes, including abnormal placentation, after repeated cesarean sections are well studied. Herein, we describe a patient with delayed hemorrhage from a uterine AVM, following dilation and curettage for a cesarean scar pregnancy. Case: A 32-year-old G3P2 presented with a cesarean scar ectopic pregnancy managed with dilation and curettage, which incurred a 1,500-ml blood loss. Within 6 weeks, she returned with 2 episodes of vaginal bleeding. Initial angiography demonstrated a highflow arteriovenous fistula, which was coiled. Vaginal hemorrhage recurred; repeat angiography demonstrated a large AVM. Gelfoam embolization of the bilateral internal iliac arteries reduced the vascularity of the AVM. The AVM's location, starting at the left lateral apex of the cesarean scar and extending into the parametrium, necessitated a radical hysterectomy. Pathologic examination revealed a placenta percreta extending into the parametrium. Conclusion: The prevalence of uterine AVMs has increased with the rise in surgical obstetrics. In patients with a failed prior interventional procedure, surgical management is necessary to prevent life-threatening hemorrhage. The location of the AVM within the abnormal uterine scar tissue requires familiarity with radical pelvic surgical techniques that are normally used in cancer surgery in order to definitively treat this delayed obstetrical complication.
\end{abstract}


Moulder et al.: The Role of Radical Surgery in the Management of Acquired Uterine Arteriovenous Malformation

\section{Introduction}

Uterine arteriovenous malformations (AVMs) are uncommon and can present as lifethreatening hemorrhage, accounting for $1-2 \%$ of all genital hemorrhage [1]. The pathogenesis of uterine AVMs may be congenital or acquired. Congenital uterine AVMs are thought to be secondary to abnormal embryologic differentiation [2]. Acquired uterine AVMs are typically a result of trauma or instrumentation, although they have also been associated with gestational trophoblastic disease, endometrial cancer, diethylstilbestrol exposure and intrauterine devices [1].

Initial imaging is done by ultrasonography, although MRI is frequently necessary to identify the extent of the lesion. Fertility-sparing treatments may include pelvic angiography and selective embolization. Herein, we present a patient who required radical surgery despite selective embolization for persistent hemorrhage secondary to an acquired uterine AVM.

\section{Case Report}

A 32-year-old G3P2012 presented with sonographic findings indicating a cesarean scar ectopic pregnancy. The lower uterine segment was $6 \mathrm{~mm}$ thick, incorporating the bladder, uterine serosa, myometrium, decidua and trophoblast. She consented to undergo diagnostic laparoscopy, dilation and curettage. Laparoscopically, the uterine serosa appeared normal. During the curettage she had a 1.5-liter postpartum hemorrhage, which stopped after placement of an intrauterine compression balloon as a tamponade.

The patient subsequently presented with profuse vaginal bleeding requiring a blood transfusion. Ultrasound revealed a multicystic structure with low-resistance arterial flow on Doppler, inseparable from the anterior lower uterine segment (fig. 1). MRI demonstrated multiple, enlarged vessels extending from the myometrium to the left broad ligament, which raised suspicion of an AVM (fig. 2). A pelvic and selective bilateral uterine arteriography was performed. The left uterine arteriography suggested the presence of a large, high-flow arteriovenous fistula (AVF), with drainage through the left internal iliac vein and left gonadal vein. The lesion was successfully embolized with coils, and an angiogram confirmed cessation of the flow. Other multiple, small AVFs supplied by both uterine arteries were considered clinically insignificant given the occlusion of the larger AVF.

Four days following coil placement, the patient experienced acute vaginal hemorrhage. Repeat angiography demonstrated a hypervascular midline mass arising from the anterior division of the internal iliac arteries bilaterally, without a dominant feeding artery. Angiographically, these represented multiple, small AVFs, which were larger and more numerous than those previously noted, consistent with acquired AVM (fig. 3). Given her recurrence of symptoms despite vascular embolization with coils, a step-wise procedure was planned: Gelfoam (Pfizer, New York, N.Y., USA) bilateral embolization of the internal iliac arteries, followed by a hysterectomy.

Gelfoam embolization of the internal iliac arteries resulted in a $40-60 \%$ reduction in vascularity of the uterine AVM (fig. 4). Grossly, the AVM originated from the prior hysterotomy site and extended into the left parametrium. The right uterine vessels and cardinal ligaments were found to have compensatory engorgement. The AVM extended into the left parametrium and left pelvic sidewall. This anatomy necessitated a radical hysterectomy for complete excision. Dissection of the potential spaces of the pelvis was undertaken, and the bladder and left ureter were dissected away from the AVM. 
Moulder et al.: The Role of Radical Surgery in the Management of Acquired Uterine Arteriovenous Malformation

\section{Surgical Technique}

A laparotomy was performed. The right and left round ligaments were ligated and the anterior and posterior leaves of the broad ligaments were incised. On the right side, the utero-ovarian ligament was transected and the right fallopian tube and ovary were preserved. On the left side, the AVM extended into the mesosalpingeal plexus and involved the left gonadal vessels. The posterior broad ligament incision was extended to the left paracolic gutter. Above the pelvic brim, the ureter was identified and separated from the gonadal vessels. The left gonadal vessels (infundibulopelvic vessels) were ligated above the extension of the AVM, and the left fallopian tube and ovary were resected en bloc with the hysterectomy specimen (see below).

The peritoneum overlying the anterior cul-de-sac and bladder flap was densely adherent to the cesarean section hysterotomy scar. The peritoneum was mobilized from the right anterior leaf of the broad ligament. The right paravesical and pararectal spaces were then opened and the right ureter was identified and freed up to the right ureteral tunnel. The bladder was sharply separated from the right side of the uterus. The right uterine artery was ligated at the level of the internal cervical os followed by the ligation of the right cardinal ligament. This mobilized the right side of the uterus down to the level of the upper one third of the vagina.

The left parametrium contained the AVM that extended from the midportion of the cesarean section hysterotomy site to the left pelvic sidewall. The left pararectal space was opened. The ureter, which had been identified at the level of the left pelvic brim, was dissected free from the medial leaf of the left broad ligament. The left uterine artery and vein were isolated at their origin off the internal iliac vessels (hypogastric vessels) and ligated. The left paravesical space was opened, the superior vesical artery was ligated, and the space of Retzius was opened; this allowed the mobilization of the left lateral edge of the bladder. The left ureteral tunnel was then fully opened, and the ureter was completely mobilized away from the AVM (fig. 5). Next, the left side of the bladder was sharply dissected away from the AVM and the hysterotomy scar. The left parametrium was ligated at the left pelvic sidewall, and the inferior aspect of the AVM was released. The uterus, cervix, left adnexa and upper one third of the vagina were then resected (fig. 6). The vaginal cuff was closed. A cell saver was used for autotransfusion. The estimated blood loss was $500 \mathrm{ml}$.

\section{Pathology}

Upon pathologic examination, the enlarged vessels and arteriovenous communications were considered part of a placenta percreta with dehiscence of hysterotomy and subinvolution of the placental implantation site, which extended into the parametrium (fig. 7).

\section{Conclusion}

This case illustrates the need for radical pelvic surgical techniques, which are used in cervical cancer surgery, to safely resect a hysterotomy scar AVM that presented with lifethreatening hemorrhage. Congenital and acquired uterine AVMs have a similar pathophysiology: AVMs are high-flow systems, with blood coursing through to the venous system under arterial pressure and flow [3]. Acquired AVMs of the uterus are characterized by single or multiple AVFs which represent the direct connection of an artery and vein without an 
intervening capillary bed, as distinct from congenital AVMs which are characterized by a nidus of poorly differentiated blood vessels connecting the arterial and venous systems. The venous system is unable to accommodate the high-pressure, high-flow state, resulting in a higher propensity for bleeding.

Arteriography can distinguish between an AVM and an AVF by demonstrating the presence or absence of a nidus, respectively. AVMs require occlusion of the nidus, whereas AVFs require occlusion of the fistulous communication or its feeding vessels. When the arterial territory distal to the AVF can withstand ischemia and the distal arteries pose no risk of retroperfusion of the AVF, the arterial feeders may be occluded with embolic particles (i.e. Gelfoam). As acquired uterine AVMs contain single or multiple AVFs, Gelfoam embolization was performed in this patient because the risk of retroperfusion was considered less important given the planned hysterectomy. The technical and clinical success rates of uterine artery embolization for traumatic AVMs are reportedly 100 and 93\%, respectively [4]. Treatment of acquired AVMs requires occlusion or resection of all arteriovenous communications to prevent further life-threatening hemorrhage. Occlusion of only one large AVF was achieved during the first interventional procedure; it became evident that the extent of the AVM was prohibitive for occlusion with particle embolization alone.

Radical hysterectomy is a procedure typically reserved for malignant disease in the parametrial tissue. The surgeon must have familiarity with the pelvic spaces and vasculature to perform the dissection and preserve anatomic function. The role of the advanced gynecologic surgeon is known in the acute setting, such as during intraoperative hemorrhage or unintended visceral injury, but should also be recognized in cases with distorted pelvic anatomy.

Abnormal implantation in the cesarean scar bed accounts for $6 \%$ of ectopic pregnancies in multiparas. More than half of these women have only had one prior cesarean delivery [5]. AVMs have been confirmed as arising from a prior cesarean scar ectopic implantation site [6]. This may result from failed obliteration of placental bed vessels in the absence of retained placental tissue. Conversely, retained villi may be found within a uterine pseudoaneurysm, with subsequent recruitment of collateral vessels [7]. This has been reported in placenta accreta and may be similar to our experience, given the pathologic diagnosis. Uterine AVMs may soon be a recognized complication of cesarean scar ectopic pregnancies. The roles of the interventional radiologist and advanced gynecologic surgeon will continue to evolve in the management of delayed obstetrical complications given the pathophysiology and anatomic challenges of the scarred pelvis.

\section{References}

1 Cura M, Martinez N, Cura A, et al: Arteriovenous malformations of the uterus. Acta Radiol 2009;50:823-829.

$\checkmark 2$ Kasznica J, Nisar N: Congenital vascular malformation of the uterus in a stillborn: a case report. Hum Pathol 1995;26:240-241.

-3 Hochman M, Adams DM, Reeves TD: Current knowledge and management of vascular anomalies, II: malformations. Arch Facial Plast Surg 2011;13:425-433.

4 Ghai S, Rajan DK, Asch MR, et al: Efficacy of embolization in traumatic uterine vascular malformations. J Vasc Interv Radiol 2003;14:1401-1408.

$>5$ Rotas MA, Haberman S, Levgur M: Cesarean scar ectopic pregnancies: etiology, diagnosis, and management. Obstet Gynecol 2006;107:1373-1381.

6 Rygh AB, Greve 0J, Fjetland L, et al: Arteriovenous malformation as a consequence of a scar pregnancy. Acta Obstet Gynecol Scand 2009;88:853-855.

7 Kwon JH, Kim GS: Obstetric iatrogenic arterial injuries of the uterus: diagnosis with US and treatment with transcatheter arterial embolization. Radiographics 2002;22:35-46. 


\section{Case Reports in Oncology}

\begin{tabular}{l|l}
\hline Case Rep Oncol 2013;6:303-310 \\
\hline DOI: $10.1159 / 000351609$ & $\begin{array}{l}\text { C 2013 S. Karger AG, Basel } \\
\text { www.karger.com/cro }\end{array}$ \\
\hline
\end{tabular}

Moulder et al.: The Role of Radical Surgery in the Management of Acquired Uterine Arteriovenous Malformation

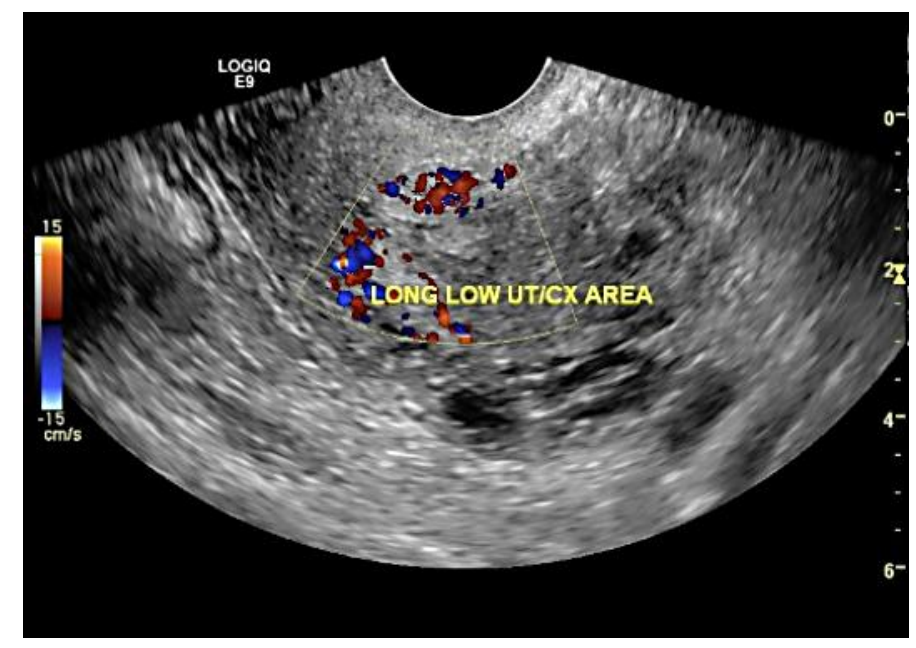

Fig. 1. Ultrasound imaging of a multicystic structure, inseparable from the anterior lower uterine segment.

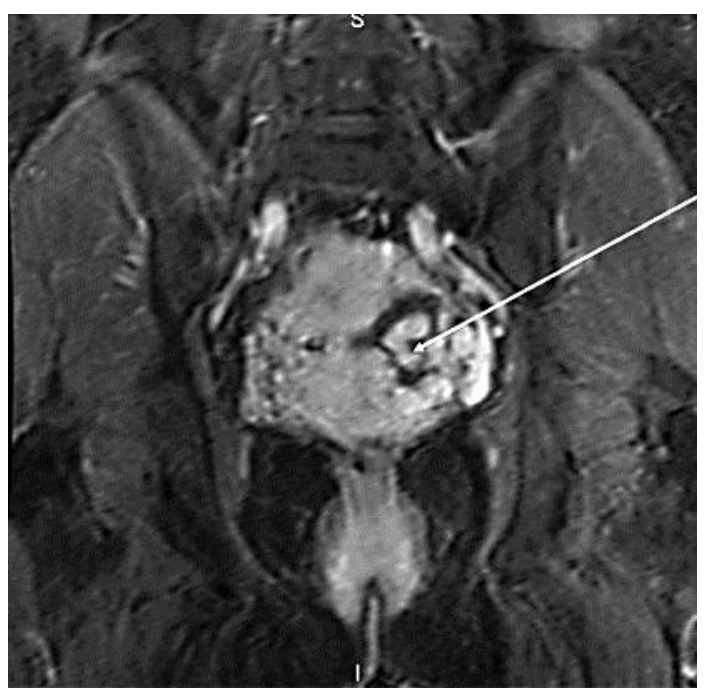

Fig. 2. MRI demonstrates multiple enlarged vessels suspicious for vascular malformation. The arrow identifies abnormal vessels. 


\section{Case Reports in Oncology}

Case Rep Oncol 2013;6:303-310

DOI: $10.1159 / 000351609$

Moulder et al.: The Role of Radical Surgery in the Management of Acquired Uterine Arteriovenous Malformation

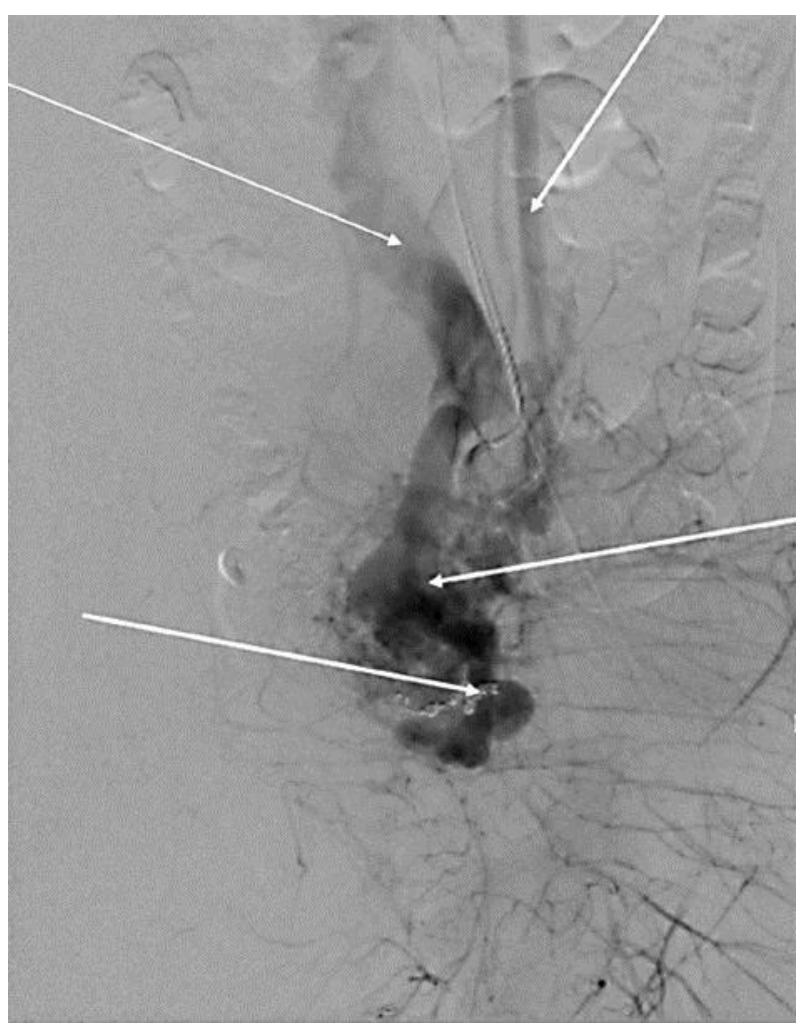

Fig. 3. Second angiography after the coiling procedure demonstrates multiple, small AVFs, consistent with an acquired AVM. Upper left arrow: draining vein. Upper right arrow: arterial inflow. Lower left arrow: coils. Lower right arrow: abnormal vessels. 


\section{Case Reports in Oncology}

\begin{tabular}{l|l}
\hline Case Rep Oncol 2013;6:303-310 \\
\hline DOI: $10.1159 / 000351609$ & $\begin{array}{l}\text { ○ 2013 S. Karger AG, Basel } \\
\text { www.karger.com/cro }\end{array}$ \\
\hline
\end{tabular}

Moulder et al.: The Role of Radical Surgery in the Management of Acquired Uterine Arteriovenous Malformation

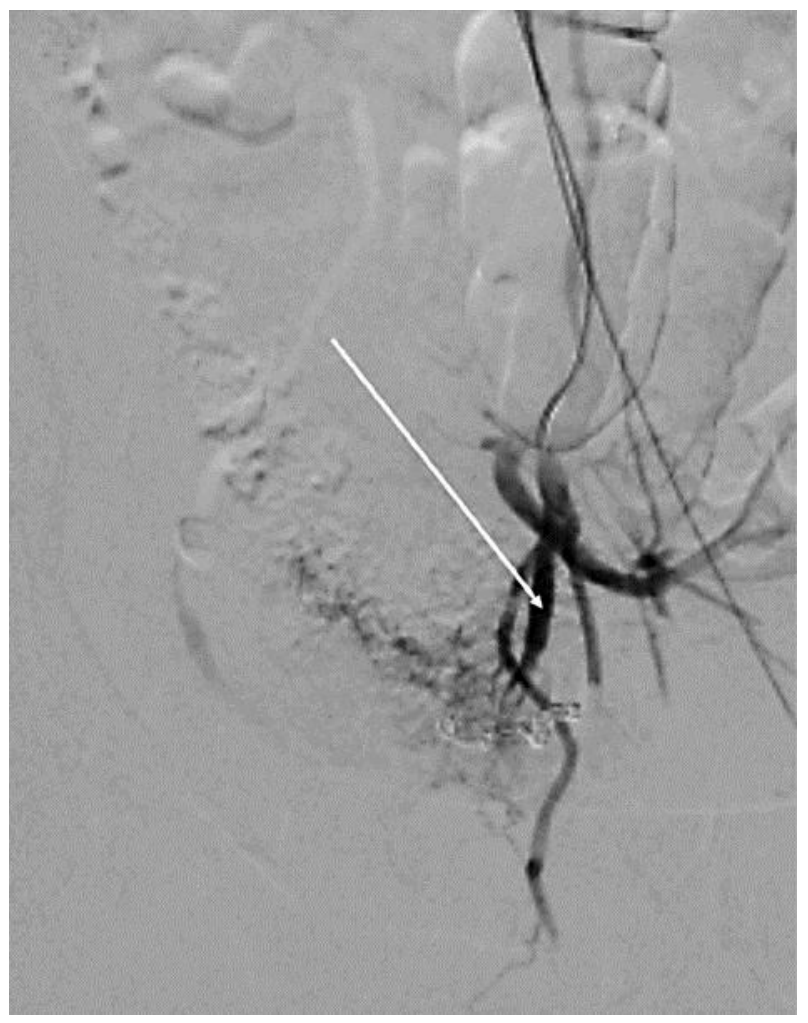

Fig. 4. Angiography following Gelfoam embolization of the internal iliac arteries demonstrates a 40-60\% reduction in vascularity of the uterine AVM. The arrow shows arterial inflow.

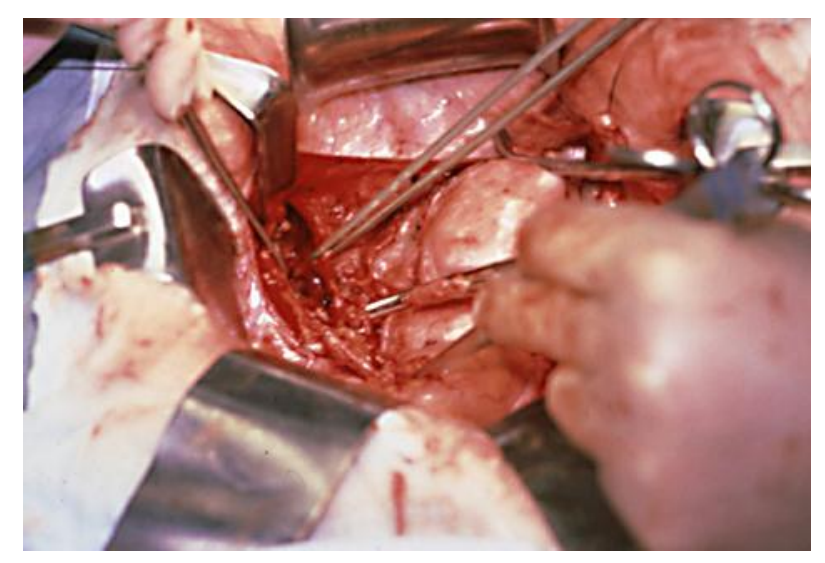

Fig. 5. Radical left pelvic sidewall dissection, opening and resection of the left parametrium and full mobilization of the left ureter (pointed to by forceps). 


\section{Case Reports in Oncology}

\begin{tabular}{l|l}
\hline Case Rep Oncol 2013;6:303-310 \\
\hline DOI: 10.1159/000351609 & $\begin{array}{l}\text { ○ 2013 S. Karger AG, Basel } \\
\text { www.karger.com/cro }\end{array}$ \\
\hline
\end{tabular}

Moulder et al.: The Role of Radical Surgery in the Management of Acquired Uterine Arteriovenous Malformation

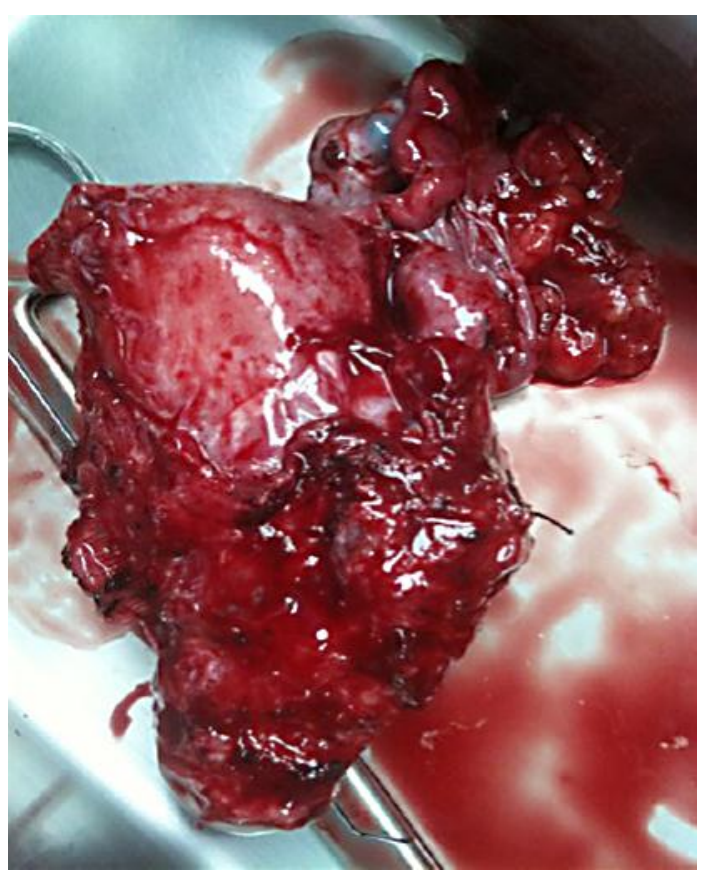

Fig. 6. Gross specimen shows arteriovenous communications, the placenta percreta and the full extension along the left parametrium.

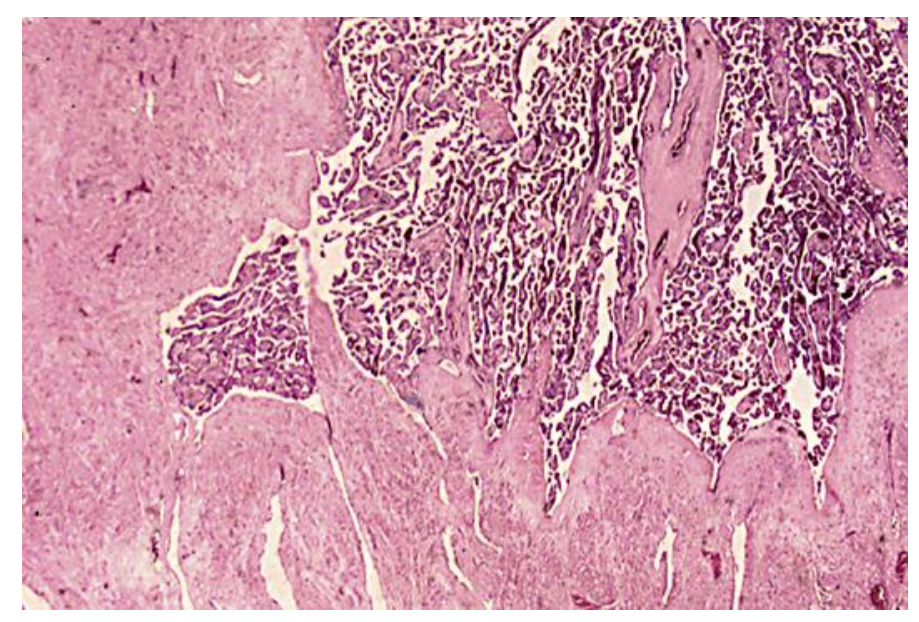

Fig. 7. Placental villi are present through the uterine wall to the serosa, in association with the cesarean section hysterotomy scar. 